\section{INTERSTELLAR SHOCK FLUORESCENCE}

\section{Ralph S. Sutherland}

Mount Stromlo and Siding Spring Observatories, Australian

National University, Canberra ACT 2601

April 1993

In this thesis, a description of the development of a new spectral synthesis code is given. The new code, MAPPINGS II, expands upon the existing code MAPPINGS (Binette 1982). It incorporates recent atomic data, published up to 1992, and provides for an extended range of plasma ionization conditions. It is capable of modeling up to 16 species in all stages of ionization. New, more rigorous methods of calculating collisional ionization and hydrogenic recombination are developed. Initial results include self-consistent non-equilibrium cooling functions for a wide range of temperatures and abundances. The cooling functions represented in Chapter 3.

In Chapter 4 the feasibility of an alternative model for extended emission line excitation in active galaxies is explored. In this model the exciting nuclear energy is transported by a mechanical flux in the form of high velocity plasma radio jets, rather than narrow, relativistically beamed, ionizing radiation.

The mechanical interaction of such a radio-jet and an extra-galactic dense cloud is invoked to account for the extended emission line filaments of Centaurus A. The resulting cloud collisions and shockwaves are modeled, and shown to be capable of producing sufficient ionizing radiation to account for the observed emission luminosity and line ratios. The model also makes important predictions about the velocity dependence and UV characteristics of the emitted spectrum. The emission spectrum of the cloudcloud collisions, which are produced in the interaction, is quite characteristic of the physical processes involved, and the model has general application wherever large-scale turbulent motions of $200-500 \mathrm{kms}^{-1}$ are encountered. This may include the narrow line regions of Seyfert galaxies and elliptical LINER galaxies.

In Chapters 5 and 6 the MAPPINGS II code is used to analyze the emission from oxygen-rich supernova remnants (OSNRs). Three remnants, N132D, Puppis A and SNR G292.0+1.8, were observed and the results are subject to an initial analysis in Chapter 5. A structural model for N132D is presented. In the case of N132D the interaction of the stellar ejecta with the local circumstellar environment is likely to be responsible for the ongoing excitation of the oxygenrich material.

In the final chapter, the observations are combined with data from the literature for other OSNRs, and an emission mechanism for the observed the oxygen-rich spectra is tested. In this model a time-dependent photoionization computation is used to calculate the emission from a shock induced R-Type ionization front propagating through an individual cloud of oxygen-rich material. The cloud shock is the product of the collision of the high velocity cloud with a density discontinuity. The comparison between the synthetic spectra and the observations is encouraging.

\section{HIGH ENERGY EMISSIONS FROM ASTROPHYSICAL OBJECTS}

\section{Anthony P Szabo}

Physics Department, University of Adelaide, Adelaide SA 5001

April 1993

In this thesis, some of the consequences of accelerating protons to high energies via shock acceleration in diffuse matter and radiation fields are examined. In particular particle production in active galactic nuclei is investigated. In the central region (or nucleus) of an active galaxy protons primarily lose energy via proton-proton interactions with the accreting matter, and pair production and pion photoproduction on the radiation field. The decay of pions produced from proton-proton and pion photoproduction interactions results in a neutrino flux from the nuclei of active galaxies. While individual AGN are not observable with the proposed high energy neutrino telescopes, the diffuse neutrino background produced by all of the active galactic nuclei may be observable.

After a brief introduction to the field of high energy astrophysics in Chapter 1, cosmic ray physics relevant to particle production in active galactic nuclei is discussed in Chapter 2. Cosmic ray propagation is discussed in terms of a transport equation similar to that suggested by Ginzburg and Syrovatskii (1964). Shock acceleration is introduced using the basic ideas of Fermi (1949) and is also discussed in the context of an infinite planar shock with a parallel magnetic field. The chapter is concluded with a description of how shock acceleration may be visualised in terms of a leaky box model.

High energy interactions (exclusive and inclusive) and decays (two and three body) are described in general terms in Chapter 3. In addition, some of the reference frames used in the modelling of high energy interactions are discussed along with a derivation of the mean path length for interaction of a particle in a relativistic gas. The techniques described in Chapter 3 are applied to pion photoproduction, pair production, proton-proton interactions and pion and muon decays in Chapter 4. Monte Carlo simulations of pion photoproduction and pair production are described along with a discussion of how proton-proton interactions may be modelled.

In Chapter 5, the techniques used for the observation of high energy neutrinos are discussed. Some of the proposed neutrino telescopes are described and their present status (or at least their status as of early in 1992) is reviewed. The method of calculating the neutrino signal in a neutrino telescope is described, and is applied to estimate the neutrino signal from SN 1987A using the model of Harding et al. (1991) for particle acceleration in the supernova remnant.

A simple model of an active galactic nucleus involving accretion onto a super-massive black hole is used in Chapter 6 to investigate the consequences of high energy particle production within the central region. Particle acceleration is assumed to take place via the first order Fermi mechanism at a shock front which forms in the accretion flow, and be supported by pressure from high energy protons. Observations of the continuum radiation from active galactic nuclei are briefly reviewed and two "generic" photon spectra are developed. Particle production by pair production, pion photoproduction and proton-proton interactions is investigated both during and after acceleration of the protons. The question of neutron escape from the core region of the active galaxy is examined and the consequences are dis- 